\title{
On Nakhleh's Metric for Reduced Phylogenetic Networks
}

\author{
Gabriel Cardona, Mercè Llabrés, Francesc Rosselló, and Gabriel Valiente
}

\begin{abstract}
We prove that Nakhleh's metric for reduced phylogenetic networks is also a metric on the classes of tree-child phylogenetic networks, semibinary tree-sibling time consistent phylogenetic networks, and multilabeled phylogenetic trees. We also prove that it separates distinguishable phylogenetic networks. In this way, it becomes the strongest dissimilarity measure for phylogenetic networks available so far. Furthermore, we propose a generalization of that metric that separates arbitrary phylogenetic networks.
\end{abstract}

Index Terms-Biology and genetics, graph algorithms, network problems.

\section{INTRODUCTION}

P HYLOGENETIC networks are explicit models of evolutionary histories that include reticulate evolutionary events like genetic recombinations, lateral gene transfers, or hybridizations. There are currently many algorithms and software tools that make it possible to reconstruct phylogenetic networks. As in the classical phylogenetic tree reconstruction setting, the assessment of phylogenetic network reconstruction methods requires the ability to compare phylogenetic networks, for instance, to compare inferred networks with either simulated networks or true phylogenies, and to evaluate the robustness of phylogenetic network reconstruction algorithms when adding new species [16], [21]. This has led to an increasing interest in the definition of dissimilarity measures for the comparison of phylogenetic networks, and their implementation in software packages.

Apart from the trivial metric (two networks are at distance 0 if they are isomorphic and at distance 1 otherwise), which gives no information on the degree of dissimilarity of a pair of networks (see [17]), these dissimilarity measures include the bipartitions, or Robinson-Foulds, metric [1], which satisfies the axioms of metrics [9] on the classes of regular networks [1] and of tree-child time consistent phylogenetic networks [7]; the tripartitions metric [16], which satisfies the axioms of metrics on the class of tree-child time consistent phylogenetic networks [7]; the $\mu$-distance [8], which is a metric on the classes of all tree-child phylogenetic networks [8] and semibinary tree-sibling time consistent phylogenetic networks [3]; the triplets metric, which is a metric on the class of tree-child time consistent phylogenetic networks [5]; and a nodal metric that is again a metric on the class of tree-child time consistent phylogenetic networks [5], [4].

- G. Cardona, M. Llabrés, and F. Rosselló are with the Department of Mathematics and Computer Science, University of the Balearic Islands, E-07122 Palma de-Mallorca, Spain.

E-mail: \{gabriel.cardona, merce.llabres, cesc.rossello\}@uib.es.

- G. Valiente is with the Algorithms, Bioinformatics, Complexity and Formal Methods Research Group, Technical University of Catalonia, E-08034 Barcelona, Spain. E-mail: valiente@lsi.upc.edu.

Manuscript received 2 Jan. 2009; revised 2 Mar. 2009; accepted 5 Mar. 2009; published online 9 Mar. 2009

For information on obtaining reprints of this article, please send e-mail to: tcbb@computer.org, and reference IEEECS Log Number TCBB-2009-01-0002. Digital Object Identifier no. 10.1109/TCBB.2009.33.
Nakhleh has recently proposed a dissimilarity measure for the comparison of phylogenetic networks [17] that can be computed in time polynomial in the size of the networks and proved that it satisfies the separation axiom of metrics (zero distance means isomorphism) on the class of all reduced phylogenetic networks in the sense of [16], and hence, that it is a metric on this class of networks. In this note, we complement and generalize Nakhleh's work in three directions. On the one hand, we prove a stronger result: namely, that for this dissimilarity measure, zero distance implies indistinguishability up to reduction in the sense of [16], a goal that had already been unsuccessfully pursued by Moret et al. in [16]. In this way, and to the best of our knowledge, Nakhleh's dissimilarity measure turns out to be the first nontrivial one that separates distinguishable networks. On the other hand, we show that this dissimilarity measure is a metric on several classes of phylogenetic networks and related (multi-)labeled DAGs: namely, on the classes of tree-child phylogenetic networks, semibinary tree-sibling time consistent phylogenetic networks, and multilabeled phylogenetic trees, including, for instance, area cladograms [13]. Adding this to the aforementioned fact, previously proved by Nakhleh, that it is a metric on the class of all reduced networks, it turns out that his latest dissimilarity measure for phylogenetic networks has the strongest separation power among all metrics defined so far. Finally, we show that a slight modification of this measure yields a metric on the whole space of all phylogenetic networks. It is not known whether this last metric can be computed in polynomial time, although the chances are that it is not possible because, on the class of tree-sibling time consistent phylogenetic networks, the isomorphism problem is polynomially equivalent to the graph isomorphism problem [6].

\section{Preliminaries}

\subsection{Notations}

Let $N=(V, E)$ be a finite directed acyclic graph (DAG). We say that a node $v \in V$ is a child of $u \in V$ if $(u, v) \in E$; we also say then that $u$ is a parent of $v$. Two children of a same parent are said to be sibling of each other. A leaf is a node without children. A node that is not a leaf is called internal. We say that a node is a tree node when it has at most one 
parent, and it is a hybrid node when it has more than one parent. A DAG is semibinary when all its hybrid nodes have exactly two parents and one child, without any restriction on the number of children of its tree nodes. A DAG is rooted when it has only one root: a node without parents.

A path in $N=(V, E)$ is a sequence of nodes $\left(v_{0}, v_{1}, \ldots, v_{k}\right)$ such that $\left(v_{i-1}, v_{i}\right) \in E$ for all $i=1, \ldots, k$. We call $v_{0}$ the origin of the path, $v_{1}, \ldots, v_{k-1}$ its intermediate nodes, $v_{k}$ its end, and $k$ its length; a path is nontrivial when its length is larger than 0 . We denote by $u \rightsquigarrow v$ any path with origin $u$ and end $v$ and whenever there exists a path $u \rightsquigarrow v$, we say that $v$ is a descendant of $u$ and $u$ is an ancestor of $v$ : if the path $u \rightsquigarrow v$ is nontrivial, we say that $v$ is a proper descendant of $u$ and $u$ is a proper ancestor of $v$.

The height $h(v)$ of a node $v$ in a DAG $N$ is the largest length of a path from $v$ to a leaf. The absence of cycles implies that the nodes of a DAG can be stratified by means of their heights: the leaves are the nodes of height 0 and, for every $m \geq 1$, the nodes of height $m$ are those internal nodes with all their children of height smaller than $m$ and at least one child of height exactly $m-1$.

Let $S$ be a nonempty finite set, whose elements are called taxa and represent species or other Operational Taxonomic Units; unless otherwise stated, for simplicity, we shall always take as $S$ the set of positive integers $\{1, \ldots, n\}$, with $n=|S|$. A phylogenetic network on a set $S$ of taxa is an $S$-DAG, that is, a rooted DAG whose leaves are bijectively labeled with elements of $S$. A phylogenetic tree is a phylogenetic network without hybrid nodes. We shall always identify, usually without any further notice, each leaf of a phylogenetic network with its label. Two phylogenetic networks $N, N^{\prime}$ are isomorphic, in symbols $N \cong N^{\prime}$, when they are isomorphic as directed graphs and the isomorphism sends each leaf of $N$ to the leaf with the same label in $N^{\prime}$.

A phylogenetic network $N=(V, E)$ is said to be tree-child when every internal node has some child that is a tree node, tree-sibling when every hybrid node has some sibling that is a tree node, and time consistent when it allows a mapping

$$
\tau: V \rightarrow \mathbb{N}
$$

such that, for every $\operatorname{arc}(u, v) \in E, \tau(u)<\tau(v)$ if $v$ is a tree node and $\tau(u)=\tau(v)$ if $v$ is a hybrid node. The biological meaning of these conditions has been discussed in [2], [3], [8], [16].

For every node $u$ of a phylogenetic network $N=(V, E)$, let $C(u)$ be the set of all its descendants in $N$ and $N(u)$ the subgraph of $N$ induced by $C(u)$ : It is still a phylogenetic network, with root $u$ and leaves labeled in the subset $C_{L}(u) \subseteq S$ of labels of the leaves that are descendants of $u$. We shall call $N(u)$ the rooted subnetwork of $N$ generated by $u$, and the set of leaves $C_{L}(u)$ the cluster of $u$.

A clade of a phylogenetic network $N$ is a rooted subnetwork of $N$ all whose nodes are tree nodes in $N$ (and, in particular, it is a phylogenetic tree).

Let $S$ be again a finite set of labels and $\mathcal{P}^{+}(S)$ the set of its nonempty subsets. A (rooted) multilabeled phylogenetic tree (an MUL-tree, for short) over $S$ is a rooted tree whose leaves are labeled with elements of $\mathcal{P}^{+}(S)$. In particular, two leaves in an MUL-tree may share one or more labels, and they may even be labeled with the same subset of $S$. Beside being used in the reconstruction of phylogenetic networks [14], MUL-trees include as special cases area cladograms [13] and tangled trees [18].

\subsection{Multisets and Metrics}

Let $\mathcal{C}$ be a class endowed with a notion of isomorphism $\cong$; for instance, the class of all phylogenetic networks on a given set of taxa. A metric on $\mathcal{C}$ is a mapping

$$
d: \mathcal{C} \times \mathcal{C} \rightarrow \mathbb{R}
$$

satisfying the following axioms: for every $A, B, C \in \mathcal{C}$,

1. Nonnegativity: $d(A, B) \geq 0$,

2. Separation: $d(A, B)=0$ if and only if $A \cong B$,

3. Symmetry: $d(A, B)=d(B, A)$,

4. Triangle inequality: $d(A, C) \leq d(A, B)+d(B, C)$.

A finite multiset of elements of a set $X$ is a mapping $M$ : $X \rightarrow \mathbb{N}$ such that its support $\{x \in X \mid M(x) \neq 0\}$ is finite. If the support of a finite multiset $M: X \rightarrow \mathbb{N}$ is $\left\{x_{1}, \ldots, x_{k}\right\}$, then this multiset can be understood as a (sort of) set consisting of $M\left(x_{i}\right)$ copies of $x_{i}$, for every $i=1, \ldots, k$; in this context, $M(x)$ is called the multiplicity of $x \in X$ in this multiset, and this multiplicity is 0 when $x$ does not belong to the support.

The cardinal $|M|$ of a finite multiset $M$ of elements of $X$ is simply the sum of the multiplicities of the elements:

$$
|M|=\sum_{x \in X} M(x)
$$

The symmetric difference of two finite multisets $M_{1}, M_{2}$ of elements of a set $X$ is the finite multiset:

$$
\begin{aligned}
M_{1} \triangle M_{2}: X & \rightarrow \mathbb{N}, \\
x & \mapsto\left|M_{1}(x)-M_{2}(x)\right| .
\end{aligned}
$$

Thus, if an element of $X$ has multiplicity $m_{1}$ in $M_{1}$ and $m_{2}$ in $M_{2}$, then it has multiplicity $\left|m_{1}-m_{2}\right|$ in $M_{1} \triangle M_{2}$.

Given a set $X$, we shall denote by $\mathcal{M}(X)$ the class of all finite multisets of elements of $X$. The mapping

$$
\begin{aligned}
d: \mathcal{M}(X) \times \mathcal{M}(X) & \rightarrow \mathbb{R}, \\
\left(M_{1}, M_{2}\right) & \mapsto\left|M_{1} \triangle M_{2}\right|,
\end{aligned}
$$

which associates to each pair of finite multisets the cardinal of their symmetric difference, is a metric on $\mathcal{M}(X)$, taking as notion of isomorphism the equality of multisets; this metric is called the symmetric difference metric on $\mathcal{M}(X)$ (see, for instance, $[9$, p. 25] for the general version on a measure space). Since the condition of being a metric is not affected by the multiplication by an scalar factor, $\frac{1}{2} d$ is also a metric on $\mathcal{M}(X)$.

We shall use several times, usually without any further notice, the following easy result.

Proposition 1. Let $F: \mathcal{C} \rightarrow \mathcal{M}(X)$ be a mapping such that if $A \cong B$, then $F(A)=F(B)$. Then, the mapping

$$
\begin{aligned}
d_{F}: \mathcal{C} \times \mathcal{C} & \rightarrow \mathbb{R} \\
(A, B) & \mapsto \frac{1}{2}|F(A) \triangle F(B)|
\end{aligned}
$$

is a metric on $\mathcal{C}$ if, and only if, it satisfies the following condition:

- If $F(A)=F(B)$, then $A \cong B$.

Proof. Notice that $d_{F}(A, B)=\frac{1}{2} d(F(A), F(B))$. Then, the nonnegativity, symmetry, and triangle inequality axioms 
for $d_{F}$ are derived from the corresponding properties of $\frac{1}{2} d$, without any further assumption. As far as the separation axiom goes, if $A \cong B$, then $F(A)=F(B)$, and hence, $d_{F}(A, B)=0$, also without any further assumption on $F$. The converse implication in the separation axiom says

$$
|F(A) \triangle F(B)|=0 \text { implies } A \cong B,
$$

and since (by the separation axiom for the symmetric difference metric) $|F(A) \triangle F(B)|=0$ is equivalent to $F(A)=F(B)$, it is clear that this remaining condition is equivalent to the condition given in the statement.

\subsection{The $\mu$-Distance}

Let $N=(V, E)$ be a phylogenetic network on $S=\{1, \ldots$, $n\}$. For every node $v \in V$ and for every $i=1, \ldots, n$, let $m_{i}(v)$ denote the number of different paths from $v$ to the leaf $i$. The path-multiplicity vector, or $\mu$-vector, for short, of $v \in V$ is

$$
\mu(v)=\left(m_{1}(v), \ldots, m_{n}(v)\right) .
$$

The $\mu$-representation of $N$ is the multiset

$$
\mu(N)=\{\mu(v) \mid v \in V\} .
$$

The $\mu$-distance between a pair of phylogenetic networks $N_{1}$ and $N_{2}$ on the same set $S$ of taxa is

$$
d_{\mu}\left(N_{1}, N_{2}\right)=\frac{1}{2}\left|\mu\left(N_{1}\right) \triangle \mu\left(N_{2}\right)\right| .
$$

This $\mu$-distance is known to be a metric on several welldefined classes of phylogenetic networks. More specifically, we have the following result (and then, Proposition 1 applies).

Theorem 1. Let $N_{1}$ and $N_{2}$ be two phylogenetic networks on the same set $S$ of taxa. Assume that one of the following two conditions holds:

1. $N_{1}$ and $N_{2}$ are both tree-child, or

2. $N_{1}$ and $N_{2}$ are both semibinary, tree-sibling, and time consistent.

Then, $\mu\left(N_{1}\right)=\mu\left(N_{2}\right)$ implies $N_{1} \cong N_{2}$.

For a proof of the case 1, see [8], and for the case 2, see [3].

\subsection{Moret et al.'s Reduction Process}

Let $N=(V, E)$ be a phylogenetic network on a set $S$ of taxa. Two nodes in $N$ are said to be convergent when they have the same cluster. The removal of sets of convergent nodes is the basis of the following reduction procedure introduced in [16].

Let $N=(V, E)$ be a phylogenetic network on $S$. If $N$ does not contain any pair of convergent nodes (for instance, if it is a phylogenetic tree), then the reduction procedure does nothing. Otherwise:

1. For every clade $T$ of $N$, with root $r_{T}$ :

- Add a new node $h_{T}$ between $r_{T}$ and its only parent.

- Label $h_{T}$ with some symbol representing the clade $T$.

- Remove $r_{T}$ and its descendants so that $h_{T}$ becomes a leaf: we shall call it a symbolic leaf.
After this step, the resulting phylogenetic network $N^{*}$ has two kinds of leaves: symbolic, which replace clades, and hybrid, which did not belong to any clade in $N$ (the reconstructible phylogenetic networks considered in [16] could not contain hybrid leaves, but they can be handled without any problem by the reduction procedure).

2. All internal nodes that are convergent in $N$ with some other node are removed from $N^{*}$, and all internal nodes of $N^{*}$ that are descendant of some removed node are also removed.

3. For every remaining node $x$ in $N^{*}$ that was a parent of a node $v$ that has been removed in step 2, add a new arc from $x$ to every (hybrid or symbolic) leaf that was a descendant of $v$ in $N^{*}$, if such an arc does not exist yet.

The resulting DAG contains no set of convergent nodes, because any pair of convergent nodes in it would have already been convergent in $N$.

4. For every symbolic leaf $h_{T}$, unlabel it and append to it the corresponding clade $T$, with an arc from $h_{T}$ to $r_{T}$.

5. Replace every node with only one parent and one child by an arc from its parent to its only child.

Since the DAG resulting from step 3 contains no pair of convergent nodes, it contains no node with only one child. Therefore, the only possible nodes with only one parent and one child after step 4 are those that were symbolic leaves with only one parent. These are the only nodes that have to be removed in this step.

Notice that the effect of steps 4 and 5 is not exactly the replacement of each symbolic leaf by the corresponding clade: the symbolic leaf $h_{T}$ survives after step 5 if it has more than one incoming arc, and in this case, the clade $T$ is appended to $h_{T}$, instead of replacing it.

The output of this procedure applied to a phylogenetic network $N$ on $S$ is a (not necessarily rooted) leaf-labeled DAG, called the reduced version of $N$ and denoted by $R(N)$.

Example 1. Let us compute the reduced version of the phylogenetic network $N$ represented in Fig. $1{ }^{1}$ The subtree rooted at $d$, with leaves 1 and 2, is a clade, and each one of the other leaves forms a clade by itself. The graph $N^{*}$ obtained after step 1 is depicted in Fig. 2a, where the symbolic leaves that replace clades are represented by dashed circles.

The maximal sets of convergent nodes in $N$ are

$$
\{b, c\},\{A, B, e, h\},\{C, 3\},\{D, 5\} .
$$

So, in step 2, we remove the nodes $b, c, e, h, A, B, C, D$, as well as all intermediate nodes in paths from them to symbolic leaves: this also removes the nodes $f, g$. So, the only internal nodes that remain after step 2 are $r$ and $a$. This yields the graph depicted in Fig. $2 b$.

In step 3, we add new arcs from $r$ and $a$ to the symbolic leaves that were descendant of removed descendants of them. This yields the graph in Fig. 2c.

In step 4, we append again to each symbolic leaf the clade it represented, and we unlabel the symbolic leaves: see Fig. 2d.

1. Henceforth, in graphical representations of DAGs, and in particular of phylogenetic networks, we shall represent hybrid nodes by squares and tree nodes by circles. 


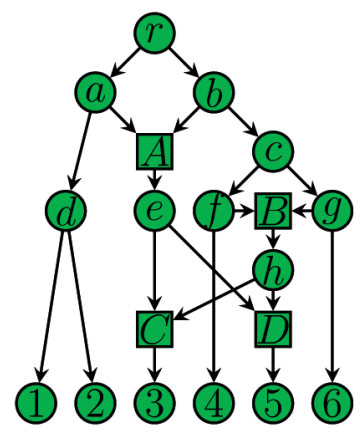

Fig. 1. The phylogenetic network $N$ in Example 1.

Finally, in step 5, the parents of the node $d$ and of the leaves 4 and 6 are removed and replaced by arcs $(a, d)$, $(r, 4)$, and $(r, 6)$, respectively. The parents of leaves 3 and 5 remain, and they are hybrid in the resulting reduced network $R(N)$, which is given in Fig. 2e.

A phylogenetic network $N$ is reduced when $R(N)=N$. From the given description of reduction procedure, it is easy to deduce that a phylogenetic network is reduced if, and only if, every pair of convergent nodes in it consists of a hybrid node of out-degree 1 and with all its proper descendants of tree type (thus forming a clade), and its only child. In particular, if we impose that all hybrid nodes in a phylogenetic network have out-degree 1 , as it is done, for instance, in reconstructible networks in the sense of [16], then a reduced network cannot contain any hybrid node that is a proper descendant of another hybrid node.

Two networks $N_{1}$ and $N_{2}$ are said to be indistinguishable when they have isomorphic reduced versions, that is, when $R\left(N_{1}\right) \cong R\left(N_{2}\right)$. Moret et al. argue in [16, p. 19] that for reconstructible phylogenetic networks, this notion of indistinguishability (isomorphism after simplification) is more suitable than the existence of an isomorphism between the original networks.

\section{Nakhleh'S Metric $m$}

In this section, we describe the dissimilarity measure $m$ introduced by Nakhleh [17]. After recalling Nakhleh's definition, we provide an alternative definition, as the cardinal of the symmetric difference of certain representations of the networks, which allows simpler proofs of the new results reported in this paper.

Nakhleh begins by defining the following equivalence of nodes in pairs of phylogenetic networks.

Definition 1. Let $N_{1}=\left(V_{1}, E_{1}\right)$ and $N_{2}=\left(V_{2}, E_{2}\right)$ be a pair of phylogenetic networks (not necessarily different). Two nodes $u \in V_{1}$ and $v \in V_{2}$ are equivalent, in symbols $u \equiv v$, when:

- $\quad u$ and $v$ are both leaves labeled with the same taxon, or

- for some $k \geq 1$, node $u$ has exactly $k$ children $u_{1}, \ldots, u_{k}$, node $v$ has exactly $k$ children $v_{1}, \ldots, v_{k}$, and possibly, after reordering, $u_{i} \equiv v_{i}$ for every $i=1, \ldots, k$.

For every node $v$ in a phylogenetic network, let $\kappa(v)$ be the number of nodes in this network that are equivalent to it.

Then, he defines his dissimilarity measure by comparing the cardinals of equivalence classes of nodes in pairs of phylogenetic networks.

Definition 2. Let $N_{1}$ and $N_{2}$ be a pair of phylogenetic networks on the same set $S$ of taxa, and let $U\left(N_{1}\right)$ and $U\left(N_{2}\right)$ be

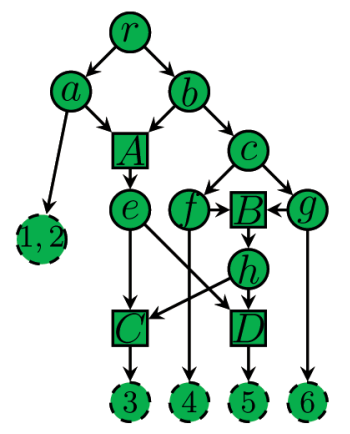

(a)

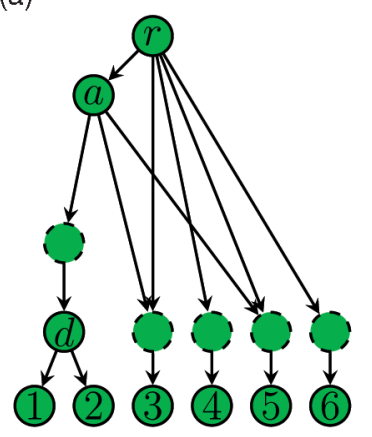

(d)

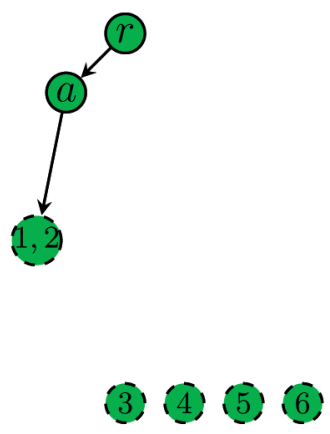

(b)

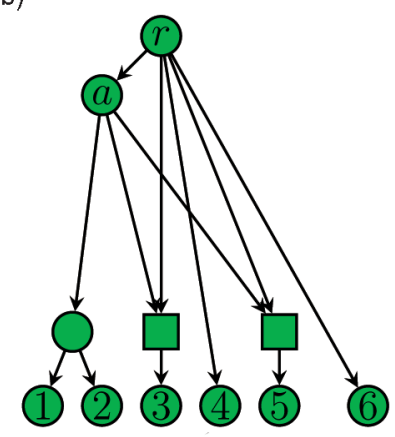

(e)

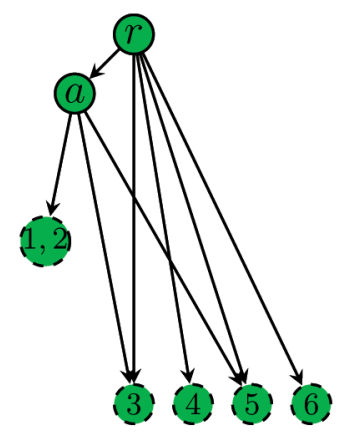

(c)

Fig. 2. The resulting DAGs after the different steps in the reduction process applied to $N$. 
TABLE 1

Nested Labels of the Nodes of the Phylogenetic Network $N$ in Fig. 1

\begin{tabular}{|l|l|}
\hline 1 & $\{1\}$ \\
\hline 2 & $\{2\}$ \\
\hline 3 & $\{3\}$ \\
\hline 4 & $\{4\}$ \\
\hline 5 & $\{5\}$ \\
\hline 6 & $\{6\}$ \\
\hline$d$ & $\{\{1\},\{2\}\}$ \\
\hline$C$ & $\{\{3\}\}$ \\
\hline$D$ & $\{\{5\}\}$ \\
\hline$e$ & $\{\{\{3\}\},\{\{5\}\}\}$ \\
\hline$h$ & $\{\{\{3\}\},\{\{5\}\}\}$ \\
\hline$A$ & $\{\{\{\{3\}\},\{\{5\}\}\}\}$ \\
\hline$B$ & $\{\{\{\{3\}\},\{\{5\}\}\}\}$ \\
\hline$f$ & $\{\{4\},\{\{\{\{3\}\},\{\{5\}\}\}\}\}$ \\
\hline$g$ & $\{\{\{\{\{3\}\},\{\{5\}\}\}\},\{6\}\}$ \\
\hline$c$ & $\{\{\{\{\{\{3\}\},\{\{5\}\}\}\},\{6\}\},\{\{4\},\{\{\{\{3\}\},\{\{5\}\}\}\}\}\}$ \\
\hline$a$ & $\{\{\{1\},\{2\}\},\{\{\{\{3\}\},\{\{5\}\}\}\}\}$ \\
\hline$b$ & $\{\{\{\{\{3\}\},\{\{5\}\}\}\},\{\{\{\{\{\{3\}\},\{\{5\}\}\}\},\{6\}\},\{\{4\},\{\{\{\{3\}\},\{\{5\}\}\}\}\}\}\}$ \\
\hline$r$ & $\{\{\{\{1\},\{2\}\},\{\{\{\{3\}\},\{\{5\}\}\}\}\}$, \\
\hline & \\
\hline
\end{tabular}

maximal sets of nonequivalent nodes in them. For every $v_{1} \in U\left(N_{1}\right)$, let

$\delta\left(v_{1}\right)=\left\{\begin{array}{c}\kappa\left(v_{1}\right), \\ \quad \text { if no node in } U\left(N_{2}\right) \text { is equivalent to } v_{1}, \\ \max \left\{0, \kappa\left(v_{1}\right)-\kappa\left(v_{1}^{\prime}\right)\right\}, \\ \quad \text { if } v_{1}^{\prime} \in U\left(N_{2}\right) \text { is equivalent to } v_{1} .\end{array}\right.$

The value $\delta\left(v_{2}\right)$, for every $v_{2} \in U\left(N_{2}\right)$, is defined in a similar way.

Then, let

$$
m\left(N_{1}, N_{2}\right)=\frac{1}{2}\left(\sum_{v_{1} \in U\left(N_{1}\right)} \delta\left(v_{1}\right)+\sum_{v_{2} \in U\left(N_{2}\right)} \delta\left(v_{2}\right)\right) .
$$

To introduce our version of this metric, we define first a nested labeling of the nodes of a phylogenetic network.

Definition 3. Let $N=(V, E)$ be a phylogenetic network on a set $S$ of taxa. The nested label $\ell(v)$ of a node $v$ of $N$ is defined by induction on $h(v)$ as follows:

- If $h(v)=0$, that is, if $v$ is a leaf, then $\ell(v)$ is the singleton consisting of its label.

- If $h(v)=m>0$, then all its children $v_{1}, \ldots, v_{k}$ have height smaller than $m$, and hence, they have been already labeled: then, $\ell(v)$ is the multiset of their nested labels:

$$
\ell(v)=\left\{\ell\left(v_{1}\right), \ldots, \ell\left(v_{k}\right)\right\} .
$$

Notice that the nested label of a node is, in general, a nested multiset (a multiset of multisets of multisets of ...), hence its name. Moreover, the height of a node $u$ is the highest level of nesting of a leaf in $\ell(u)$ minus 1 , and the cluster of $u$ consists of the taxa appearing in $\ell(u)$.

Example 2. Table 1 gives the nested labels of the nodes of the phylogenetic network depicted in Fig. 1, sorted by their height.

We shall say that a nested label $\ell(v)$ is contained in a nested label $\ell(u)$, in symbols $\ell(v) \preccurlyeq \ell(u)$, when $\ell(v)$ is the nested label of a descendant of $u$. Notice that the fact that $\ell(v)$ is contained in $\ell(u)$ does not imply that $v$ is a descendant of $u$ : several instances of this fact can be detected in the network represented in Fig. 1. Notice, moreover, that $\ell(v) \in$ $\ell(u)$ if, and only if, $\ell(v)$ is the nested label of a child of $u$.

Nakhleh's equivalence relation is easily characterized in terms of nested labels.

Proposition 2. Let $N_{1}=\left(V_{1}, E_{1}\right)$ and $N_{2}=\left(V_{2}, E_{2}\right)$ be a pair of phylogenetic networks (not necessarily different) labeled in a set $S$. For every $u \in V_{1}$ and $v \in V_{2}, u \equiv v$ if, and only if, $\ell(u)=\ell(v)$.

Proof. We prove the equivalence by induction on the height of one of the nodes, say $u$.

If $h(u)=0$, then it is a leaf and $\ell(u)$ is the one-element set consisting of its label. Thus, in this case, $u \equiv v$ if, and only if, $v$ is the leaf of $N_{2}$ with the same label as $u$, and 
$\ell(u)=\ell(v)$ if, and only if, $v$ is the leaf of $N_{2}$ with the same label as $u$, too.

Consider now the case when $h(u)=m>0$ and assume that the thesis holds for all nodes $u^{\prime} \in V_{1}$ of height smaller than $m$. Let $u_{1}, \ldots, u_{k}$ be the children of $u$. Then:

- $\quad u \equiv v$ if and only if $v$ has exactly $k$ children and they can be ordered $v_{1}, \ldots, v_{k}$ in such a way that $u_{i} \equiv v_{i}$ for every $i=1, \ldots, k$.

- $\quad \ell(u)=\ell(v)$ if and only if $v$ has exactly $k$ children and the multiset of their nested labels is equal to the multiset of nested labels of $u_{1}, \ldots, u_{k}$, which means that $v$ children can be ordered $v_{1}, \ldots, v_{k}$ in such a way that $\ell\left(u_{i}\right)=\ell\left(v_{i}\right)$ for every $i=1, \ldots, k$.

Since, by induction, the children of $u$ satisfy the thesis, it is clear that $u \equiv v$ is equivalent to $\ell(u)=\ell(v)$.

Thus, we can rewrite Nakhleh's dissimilarity measure in terms of nested labels.

Definition 4. For every $S-D A G N$, the nested labels representation of $N$ is the multiset $\Upsilon(N)$ of nested labels of its nodes (where each nested label appears with multiplicity the number of nodes having it).

Proposition 3. For every pair $N_{1}, N_{2}$ of phylogenetic networks over the same set $S$ of taxa

$$
m\left(N_{1}, N_{2}\right)=\frac{1}{2}\left|\Upsilon\left(N_{1}\right) \triangle \Upsilon\left(N_{2}\right)\right|,
$$

where $\triangle$ denotes the symmetric difference of multisets.

Proof. Let $N_{1}, N_{2}$ be a pair of phylogenetic networks over the same set $\mathrm{S}$ of taxa, and $U\left(N_{1}\right), U\left(N_{2}\right)$ maximal sets of nonequivalent nodes in them. Since the equivalence of nodes is synonymous of having the same nested labels, it is clear that, for every $i=1,2, \Upsilon\left(N_{i}\right)$ is the multiset consisting of $\kappa(v)$ copies of $\ell(v)$, for each $v \in U\left(N_{i}\right)$. Then:

- If $v_{1} \in U\left(N_{1}\right)$ is not equivalent to any node in $U\left(N_{2}\right)$, then $\ell\left(v_{1}\right)$ contributes $\kappa\left(v_{1}\right)=\delta\left(v_{1}\right)$ to $\left|\Upsilon\left(N_{1}\right) \triangle \Upsilon\left(N_{2}\right)\right|$.

- If $v_{2} \in U\left(N_{2}\right)$ is not equivalent to any node in $U\left(N_{1}\right)$, then $\ell\left(v_{2}\right)$ contributes $\kappa\left(v_{2}\right)=\delta\left(v_{2}\right)$ to $\left|\Upsilon\left(N_{1}\right) \triangle \Upsilon\left(N_{2}\right)\right|$.

- If $v_{1} \in U\left(N_{1}\right)$ is equivalent to $v_{2} \in U\left(N_{2}\right)$, then $\ell\left(v_{1}\right)=\ell\left(v_{2}\right)$ contributes

$$
\begin{aligned}
& \left|\kappa\left(v_{1}\right)-\kappa\left(v_{2}\right)\right| \\
& \quad=\max \left\{0, \kappa\left(v_{1}\right)-\kappa\left(v_{2}\right)\right\}+\max \left\{0, \kappa\left(v_{2}\right)-\kappa\left(v_{1}\right)\right\} \\
& \quad=\delta\left(v_{1}\right)+\delta\left(v_{2}\right)
\end{aligned}
$$

to $\left|\Upsilon\left(N_{1}\right) \triangle \Upsilon\left(N_{2}\right)\right|$.

This implies that

$$
\left|\Upsilon\left(N_{1}\right) \triangle \Upsilon\left(N_{2}\right)\right|=\sum_{v_{1} \in U\left(N_{1}\right)} \delta\left(v_{1}\right)+\sum_{v_{2} \in U\left(N_{2}\right)} \delta\left(v_{2}\right),
$$

from where the equality $m\left(N_{1}, N_{2}\right)=\frac{1}{2}\left|\Upsilon\left(N_{1}\right) \triangle \Upsilon\left(N_{2}\right)\right|$ follows.
In the rest of this paper, we shall use the definition of $m$ provided by the last proposition.

The value $m\left(N_{1}, N_{2}\right)$ can be computed in time polynomial in the sizes of the networks $N_{1}, N_{2}$ by first obtaining a compacted representation of the nested labels in the two networks, and then, performing a simultaneous traversal of the sorted multisets of compact nested labels of the two networks [19], [20]. The compacted representation consists in replacing the nested labels of the children of each node by unique identifiers during a bottom-up traversal of the two networks, using the idea from [12] that a procedure for dynamically maintaining a global table of unique identifiers allows the compacted directed acyclic graph representation of a tree to be determined in polynomial time.

\section{The Separation Power of $m$ \\ 4.1 $m$ Separates Distinguishable Networks}

Nakhleh proved in [17, Lemma 4] the following result.

Proposition 4. Let $R\left(N_{1}\right)$ and $R\left(N_{2}\right)$ be the reduced versions of two phylogenetic networks on the same set $S$ of taxa. Then, $m\left(R\left(N_{1}\right), R\left(N_{2}\right)\right)=0$ if, and only if, $R\left(N_{1}\right) \cong R\left(N_{2}\right)$.

In this section, we extend this result by showing that $m$ separates phylogenetic networks that are distinguishable up to reduction. We would like to recall here that this was the (unaccomplished: see [7]) goal of the error metric defined in [16].

Theorem 2. Let $N_{1}$ and $N_{2}$ be two phylogenetic networks on the set $S$ of taxa. If $m\left(N_{1}, N_{2}\right)=0$, then $N_{1}$ and $N_{2}$ are indistinguishable.

Proof. Let $N_{1}=\left(V_{1}, E_{1}\right)$ and $N_{2}=\left(V_{2}, E_{2}\right)$ be two phylogenetic networks such that $\Upsilon\left(N_{1}\right)=\Upsilon\left(N_{2}\right)$. We shall prove that the reduction process of both networks modifies exactly in the same way their nested labels representations, and thus, the reduced versions $R\left(N_{1}\right)$ and $R\left(N_{2}\right)$ are also such that $\Upsilon\left(R\left(N_{1}\right)\right)=\Upsilon\left(R\left(N_{2}\right)\right)$. Then, by Proposition 4 , the latter are isomorphic.

To begin with, notice that two nodes are convergent when the sets of taxa appearing in their nested labels are the same (without taking into account nesting levels or multiplicities). In particular, $N_{1}$ and $N_{2}$ have the same sets of nested labels of convergent nodes.

Step 1 in the reduction process consists of replacing every clade by a symbolic leaf. This corresponds to remove the nested labels of the nodes belonging to clades (except their roots) and to replace, in all remaining nested labels, each nested label of a root of a clade by the label of the corresponding symbolic leaf. We must prove now that we can decide from the nested labels representations alone which are the nested labels of nodes of clades and roots of clades.

Since the clades of a phylogenetic network are subtrees, a node belonging to a clade is only equivalent to itself (if $v$ is a node of a clade and $\ell(u)=\ell(v)$, then $C_{L}(u)=C_{L}(v)$, but in this case, since $v$ is the least common ancestor of $C_{L}(v)$ in the clade it belongs, $v$ must be a descendant of $u$, and since $u$ and $v$ have the same height-because they have the same nested label-they must be the same node). In particular, a node of a clade does not share its nested label with any other node. 
Then, the nested labels of nodes $v \in V_{i}$ belonging to some clade of $N_{i}(i=1,2)$ are characterized by the following two properties: $\ell(v)$ and each one of the nested labels contained in it appear with multiplicity 1 in $\Upsilon\left(N_{1}\right)=\Upsilon\left(N_{2}\right)$ (and in particular, $v$ and its descendants are characterized by their nested labels); and $\ell(v)$ and each one of the nested labels contained in it belong at most to one nested label (this means that $v$ and its descendants are tree nodes, and in particular that the rooted subnetwork generated by $v$ is a tree consisting only of tree nodes from $N_{i}$ ). And therefore, the roots of clades of $N_{i}$ are the nodes $v$ with nested label $\ell(v)$ maximal with these properties, and the nodes of the clade rooted at $v$ are those nodes with nested labels contained in $\ell(v)$. This shows that the nested labels of roots of clades and the nested labels of nodes belonging to clades in $N_{1}$ are the same as in $N_{2}$.

So, we remove the same nested labels in $N_{1}$ and $N_{2}$ and replace the same nested labels by symbolic leaves. As a consequence, the networks resulting after this step have the same nested labels.

In step 2, all internal nodes that are convergent with some other node are removed, and all nodes other than (symbolic or hybrid) leaves that are descendant of some removed node are also removed. So, in this step, we remove the nested labels other than singletons of convergent nodes, and the nested labels other than singletons that are contained in a nested label of some convergent node (notice that if $\ell(v)$ is not a singleton and is contained in $\ell(u)$ and $u$ is convergent, then either $v$ is a descendant of $u$, and then, it has to be removed, or it is equivalent to a descendant of $u$, and then, it is convergent with this descendant and it has to be removed, too). This shows that the nested labels of the nodes removed in both networks are the same, and hence that the nested labels of the nodes that remain in both networks are also the same.

In step 3, the paths from the remaining nodes to the labels are restored. It means to replace in each remaining nested label $\ell(x)$, each maximal nested label $\ell(v) \preccurlyeq \ell(x)$ of a removed node $v$ by the singletons $\left\{s_{1}\right\},\left\{s_{2}\right\}, \ldots,\left\{s_{p}\right\}$ of the leaves appearing in $\ell(v)$, without repeating these singletons. Again, this operation only depends on the nested labels, and therefore, after this step, the resulting DAGs have the same nested labels representations.

In step 4 , clades are restored. This is simply done by replacing in the nested labels each symbolic leaf $s$ by the nested label of the root of the clade it replaced, between braces (because we append it to the node corresponding to the symbolic leaf), as well as adding the nested labels of all nodes of the clades to the nested labels representations of the networks. Since the same clades were removed in both networks and replaced by the same symbolic leaves, after this step, the resulting DAGs still have the same nested labels representations.

Finally, in step 5, the nodes with only one parent and only one child are removed. This corresponds to removing nested labels of the form $\{\{\ldots\}\}$ that are children of only one parent (that is, that belong to only one nested label), and replacing them in the nested labels containing them by the corresponding nested label $\{\ldots\}$ without the outer braces. This shows that the same nested labels are removed in both DAGs and the remaining nested labels are modified in exactly the same way.

So, at the end of this procedure, the resulting DAGs $R\left(N_{1}\right)$ and $R\left(N_{2}\right)$ have the same nested labels representations. By Proposition 4, this implies that $R\left(N_{1}\right)$ and $R\left(N_{2}\right)$ are isomorphic.

The converse implication is, of course, false: since the reduction process may remove parts with different topologies that yield differences in the nested labels representations, two phylogenetic networks with isomorphic reduced versions may have different nested labels representations.

\section{2 $m$ Refines the $\mu$-Distance}

As a direct consequence of Proposition 4, Nakhleh deduced that $m$ satisfies the separation axiom of metrics on the class of all reduced phylogenetic networks on the same set $S$ of taxa. In this section, we show two other independent classes of phylogenetic networks where $m$ satisfies this axiom. The key observation in our proofs is that $m$ refines the $\mu$-distance, in the sense of Proposition 5 below.

Lemma 1. Let $v$ be a node in a phylogenetic network $N$ on a set $S$ of taxa. For every $i \in S, m_{i}(v)$ is the number of times the label $i$ appears in $\ell(v)$.

Proof. We prove it by induction on $h(v)$. If $h(v)=0$, then $v$ is a leaf, and therefore, $m_{i}(v)=1$ if $\ell(v)=\{i\}$ and $m_{i}(v)=$ 0 if $\ell(v)=\{j\}$, for some $j \in S \backslash\{i\}$.

Assume now that the statement is true for all nodes of height at most $m-1$, and let $v$ be a node of height $m$. Let $v_{1}, \ldots, v_{k}$ be the children of $v$, all of them of height lower than $m$. Then, on the one hand, $m_{i}(v)=m_{i}\left(v_{1}\right)+\cdots+$ $m_{i}\left(v_{k}\right)$ by [8, Lemma 4], and, on the other hand, since $\ell(v)=\left\{\ell\left(v_{1}\right), \ldots, \ell\left(v_{k}\right)\right\}$, it is clear that the number of times the label $i$ appears in $\ell(v)$ is equal to the sum of the numbers of times it appears in the nested labels $\ell\left(v_{1}\right), \ldots, \ell\left(v_{k}\right)$, which is equal, by the induction hypothesis, to $m_{i}\left(v_{1}\right)+\cdots+m_{i}\left(v_{k}\right)$.

Corollary 1. Let $N_{1}=\left(V_{1}, E_{1}\right)$ and $N_{2}=\left(V_{2}, E_{2}\right)$ be phylogenetic networks on the same set $S$ of taxa. For every $v_{1} \in V_{1}$ and $v_{2} \in V_{2}$, if $\ell\left(v_{1}\right)=\ell\left(v_{2}\right)$, then $\mu\left(v_{1}\right)=\mu\left(v_{2}\right)$.

Proposition 5. Let $N_{1}=\left(V_{1}, E_{1}\right)$ and $N_{2}=\left(V_{2}, E_{2}\right)$ be two phylogenetic networks on the same set $S$ of taxa. Then, $m\left(N_{1}, N_{2}\right) \geq d_{\mu}\left(N_{1}, N_{2}\right)$.

Proof. Let us rename the nodes of $N_{1}$ and $N_{2}$ as

$$
\begin{aligned}
& V_{1}=\left\{v_{1}, v_{2}, \ldots, v_{l}, v_{l+1}, \ldots, v_{m}, \ldots, v_{s}\right\}, \\
& V_{2}=\left\{w_{1}, w_{2}, \ldots, w_{l}, w_{l+1}, \ldots, w_{m}, \ldots, w_{t}\right\},
\end{aligned}
$$

with $\left|V_{1}\right|=s$ and $\left|V_{2}\right|=t$ and $l \leq m \leq s, t$, in such a way that:

- for every $i=1, \ldots, l, \ell\left(v_{i}\right)=\ell\left(w_{i}\right)$ (and hence, by the last corollary, $\left.\mu\left(v_{i}\right)=\mu\left(w_{i}\right)\right)$, while for every $j=l+1, \ldots, s$ and $k=l+1, \ldots, t, \ell\left(v_{j}\right) \neq \ell\left(w_{k}\right)$;

- for every $i=l+1, \ldots, m, \mu\left(v_{i}\right)=\mu\left(w_{i}\right)$, while for every $j=m+1, \ldots, s$ and $k=m+1, \ldots, t$, $\mu\left(v_{j}\right) \neq \mu\left(w_{k}\right)$. 


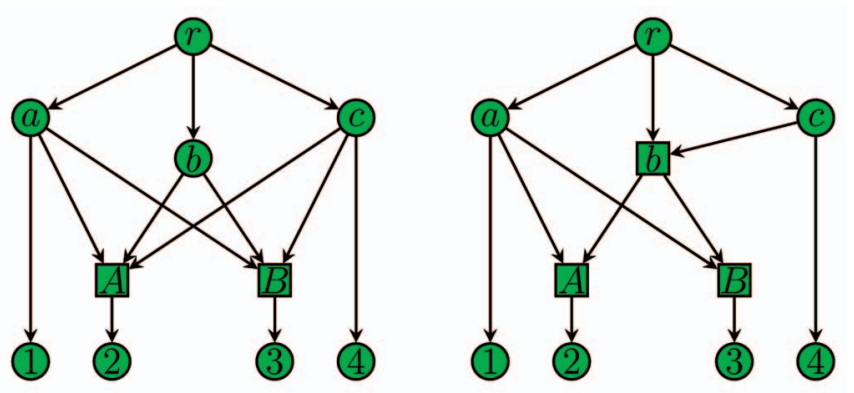

Fig. 3. Two nonisomorphic reduced phylogenetic networks at $\mu$-distance 0 .

Therefore,

$$
\begin{aligned}
\left|\Upsilon\left(N_{1}\right) \triangle \Upsilon\left(N_{2}\right)\right| & =(s-l)+(t-l) \geq(s-m)+(t-m) \\
& =\left|\mu\left(N_{1}\right) \triangle \mu\left(N_{2}\right)\right|,
\end{aligned}
$$

as we claimed.

Corollary 2. If $d_{\mu}$ satisfies the separation axiom on a class of phylogenetic networks, $m$ also satisfies it.

Proof. Let $\mathcal{N}$ be a class of phylogenetic networks such that $d_{\mu}\left(N_{1}, N_{2}\right)=0$ implies $N_{1} \cong N_{2}$ for every $N_{1}, N_{2} \in \mathcal{N}$. Let now $N_{1}, N_{2} \in \mathcal{N}$ be such that $m\left(N_{1}, N_{2}\right)=0$. Since $m\left(N_{1}, N_{2}\right) \geq d_{\mu}\left(N_{1}, N_{2}\right) \geq 0$, we conclude that $d_{\mu}\left(N_{1}\right.$, $\left.N_{2}\right)=0$ and hence, by assumption, $N_{1} \cong N_{2}$.

Combining this result with Theorem 1, we obtain the following result.

Corollary 3. Let $N_{1}$ and $N_{2}$ be two phylogenetic networks on the same set $S$ of taxa. Assume that one of the following two conditions holds:

1. $N_{1}$ and $N_{2}$ are both tree-child, or

2. $N_{1}$ and $N_{2}$ are both semibinary, tree-sibling, and time consistent.

Then, $\Upsilon\left(N_{1}\right)=\Upsilon\left(N_{2}\right)$ implies $N_{1} \cong N_{2}$.

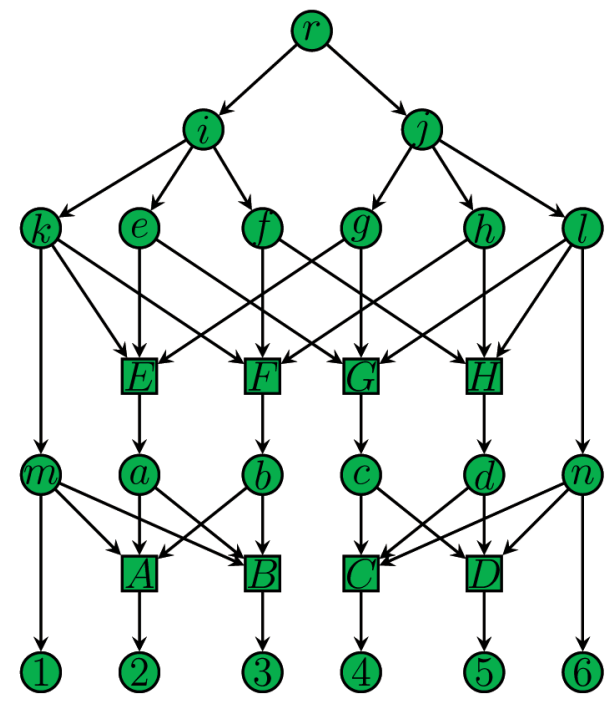

In particular, by Proposition $1, m$ is a metric on the classes of all tree-child and all semibinary, tree-sibling time consistent phylogenetic networks.

Remark 1. It is important to point out that the $\mu$-distance does not satisfy the separation axiom on the class of reduced phylogenetic networks: for instance, the reduced networks in Fig. 3 have the same $\mu$-representations, but they are not isomorphic. Therefore, Nakhleh's $m$ metric has a stronger separating power than the $\mu$-distance, in the sense that it satisfies the separation axiom in every class where $d_{\mu}$ satisfies it, and in at least one class where $d_{\mu}$ does not satisfy it.

Remark 2. It is false, in general, that if two arbitrary treesibling time consistent phylogenetic networks $N_{1}$ and $N_{2}$ on the same set $S$ of taxa are such that $m\left(N_{1}, N_{2}\right)=0$, then $N_{1} \cong N_{2}$. For instance, it is easy to check that the networks depicted in Fig. 4 have the same nested labels representations, but they are not isomorphic. Thus, Nakhleh's dissimilarity measure is not a metric on the class of all tree-sibling time consistent phylogenetic networks.

\section{3 $m$ Singles Out MUL-Trees}

The comparison of MUL-trees generalizes simultaneously the comparison of nonlabeled rooted trees (understood as MUL-trees with all their leaves labeled with the same label) and rooted phylogenetic trees (MUL-trees where each leaf has one label, and different leaves have different labels). Ganapathy et al. [13] have recently proposed two metrics for MUL-trees, an edit distance that generalizes the Robinson-Foulds distance for phylogenetic trees, and a metric based on the computation of the multilabeled analogous of a Maximum Agreement Subtree. In this section, we show that the natural generalization of Nakhleh's $m$ to MUL-trees is also a metric on the space of MUL-trees on a given set $S$ of taxa.

The definition of the nested labeling generalizes to MULtrees in a natural way as follows:

Definition 5. Let $M=(V, E)$ be an MUL-tree on a set $S$ of labels. The nested labeling $\ell(v)$ of the nodes $v$ of $N$ is defined by induction on $h(v)$ as follows:

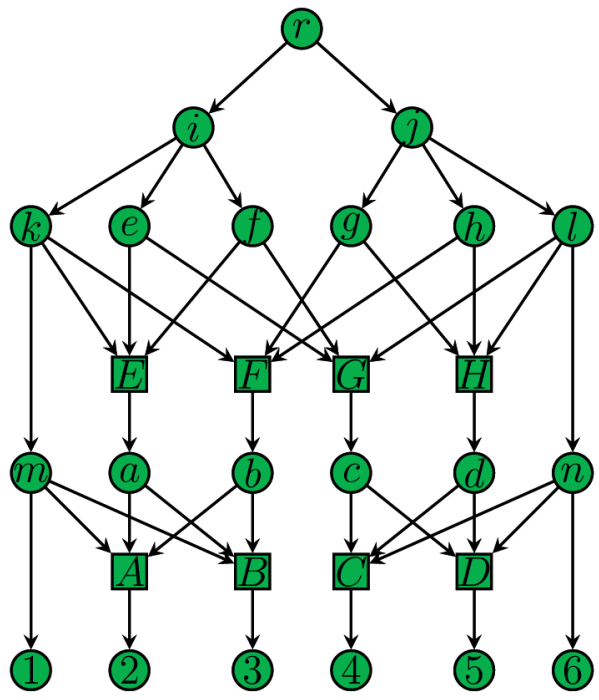

Fig. 4. These tree-sibling time consistent phylogenetic networks have the same nested labels representations, but they are not isomorphic. 
- If $h(v)=0$, that is, if $v$ is a leaf, and if the set of labels of $v$ is $S_{v} \subseteq S$, then $\ell(v)=S_{v}$.

- If $h(v)=\bar{m}>0$ and the children of $v$ are $v_{1}, \ldots, v_{k}$, then $\ell(v)$ is the multiset of their nested labels:

$$
\ell(v)=\left\{\ell\left(v_{1}\right), \ldots, \ell\left(v_{k}\right)\right\} .
$$

The nested labels representation of $M$ is the multiset $\Upsilon(M)$ of nested labels of its nodes (where each nested label appears with multiplicity the number of nodes having it).

With this definition of nested labels, Nakhleh's dissimilarity measure $m$ for MUL-trees is simply defined as in Proposition 3: half the cardinal of the symmetric difference of the nested labels representations.

Notice now that, in an MUL-tree, the nested label of a node yields, after replacing braces by parentheses, the Newick string of the subtree rooted at that node, which is a unique (up to reordering) representation of a phylogenetic tree [11, p. 590]. As it happens with Newick strings of phylogenetic trees, the nested label of the root of an MULtree characterizes the MUL-tree, up to isomorphism.

Lemma 2. Let $M_{1}$ and $M_{2}$ be two MUL-trees, and $r_{1}$ and $r_{2}$ be their respective roots. If $\ell\left(r_{1}\right)=\ell\left(r_{2}\right)$, then $M_{1} \cong M_{2}$.

Proof. We prove the statement by induction on the height $h$ of the MUL-trees: notice that the height of each $M_{i}$ is the highest level of nesting of a leaf in $\ell\left(r_{i}\right)$ minus 1 , and therefore, if both roots have the same nested labels, the MUL-trees have the same height. The case when $h=0$ is straightforward. Assume that the statement is true for pairs of MUL-trees of height lower than $h$, and that $M_{1}$ and $M_{2}$ have the same height $h$. If $\ell\left(r_{1}\right)=\ell\left(r_{2}\right)$, then both roots have the same number of children and for each child of $r_{1}$, there exists another child of $r_{2}$ with the same nested label, which implies by the induction hypothesis that the corresponding sub-MUL-trees of $M_{1}$ and $M_{2}$ rooted at both children are isomorphic. From this, it follows that $M_{1}$ and $M_{2}$ are isomorphic.

Proposition 6. Let $M_{1}$ and $M_{2}$ be two MUL-trees on the same set $S$ of labels. If $\Upsilon\left(M_{1}\right)=\Upsilon\left(M_{2}\right)$, then $M_{1} \cong M_{2}$.

Proof. If $r_{1}$ and $r_{2}$ are the roots of $M_{1}$ and $M_{2}$, respectively, then $\ell\left(r_{1}\right)$ and $\ell\left(r_{2}\right)$ are the nested labels with the highest level of nesting in $\Upsilon\left(M_{1}\right)$ and $\Upsilon\left(M_{2}\right)$, respectively, and then, these multisets being equal, it must happen that $\ell\left(r_{1}\right)=\ell\left(r_{2}\right)$. But then, by the previous lemma, $M_{1}$ and $M_{2}$ are isomorphic.

In particular, $m$ is a metric on the class of all MUL-trees labeled in a given set $S$, and it can be computed in polynomial time, because isomorphism of MUL-trees can be tested in linear time [13].

\section{A Metric for Arbitrary Phylogenetic Networks}

As Nakhleh mentions in [17], "The rationale behind the measure $m$ is that it roughly quantifies the number of rooted subnetworks that are in one but not both of the networks." As a matter of fact, if instead of nested labels of nodes, which give an incomplete description of the rooted subnetwork generated by a node, we consider the whole rooted subnetworks generated by the nodes, then we can define a true distance on the whole class of all phylogenetic networks.

Remark 3. It is clear that if $u$ and $v$ are two nodes of two phylogenetic networks $N_{1}$ and $N_{2}$, respectively (it can happen that $N_{1}=N_{2}$ ), such that the rooted subnetworks $N_{1}(u)$ and $N_{2}(v)$ generated by them are isomorphic, then $\ell(u)=\ell(v)$. But the converse implication is false: Equality of nested labels does not imply equality of rooted subnetworks. Consider, for instance, the nonisomorphic phylogenetic networks depicted in Fig. 4: It is easy to check that their roots have the same nested labels.

Definition 6. For every S-DAG $N$, let $\Sigma(N)$ be the multiset of isomorphism classes of the rooted subnetworks generated by its nodes.

Definition 7. For every pair of phylogenetic networks $N_{1}$ and $N_{2}$ on the same set $S$ of taxa, let

$$
\sigma\left(N_{1}, N_{2}\right)=\frac{1}{2}\left|\Sigma\left(N_{1}\right) \triangle \Sigma\left(N_{2}\right)\right|,
$$

where $\triangle$ denotes the symmetric difference of multisets.

Theorem 3. Let $N_{1}$ and $N_{2}$ be two phylogenetic networks on the same set $S$ of taxa. Then, $\sigma\left(N_{1}, N_{2}\right)=0$ if, and only if, $N_{1} \cong N_{2}$.

Proof. Assume that $\sigma\left(N_{1}, N_{2}\right)=0$, that is, $\Sigma\left(N_{1}\right)=\Sigma\left(N_{2}\right)$. Since each $N_{i}$ is its rooted subnetwork generated by its root, we conclude that $N_{1}$ contains a rooted subnetwork isomorphic to $N_{2}$ and $N_{2}$ contains a rooted subnetwork isomorphic to $N_{1}$. The only possibility is then that $N_{1}$ and $N_{2}$ are isomorphic (otherwise, $N_{1}$ would contain a rooted subnetwork isomorphic to it and strictly contained in it, something that in finite graphs is impossible).

The converse implication is obvious.

Example 3. It is easy to check that in each one of the networks $N_{1}$ and $N_{2}$ depicted in Fig. 4, only the root and their children generate rooted subnetworks that are not rooted subnetworks of the other network. This implies that $\sigma\left(N_{1}, N_{2}\right)=3$.

Corollary 4. The mapping $\sigma$ is a metric on the class of all phylogenetic networks on the set $S$ of taxa.

The computation of $\sigma$ has at least the same complexity as the $S$-DAG isomorphism problem (because the latter can be decided using $\sigma$ ), and isomorphism of general DAGs can be reduced to $S$-DAG isomorphism. Therefore, the problem of deciding whether $\sigma$ can be computed in polynomial time for arbitrary phylogenetic networks remains open. But if we bound the in- and out-degree of the nodes, the $S$-DAG isomorphism problem is in P [15], and therefore, $\sigma$ can be computed in polynomial time by performing a simultaneous bottom-up traversal of the two networks.

\section{CONCLUSION}

In this paper, we have complemented Nakhleh's latest proposal of a metric $m$ for phylogenetic networks [17] by showing that it separates distinguishable networks and satisfies the separation axiom on the classes of tree-child and semibinary tree-sibling time consistent phylogenetic networks as well as of multilabeled trees and, in particular, 
of area cladograms. When $m$ is applied to phylogenetic trees, it yields half the symmetric differences of the sets of (isomorphism classes of) subtrees [17], and it can be computed in time polynomial in the size of the trees.

Notice that both Nakhleh's $m$ metric and its generalization $\sigma$ satisfy the requirement, proposed by Dobson [10] in the context of metrics for phylogenetic trees, that they increase with the depth where they start to differ; this is another advantage of our general metric $\sigma$ over the trivial 0-1 metric mentioned in Section 1.

Given a set $S$ of $n \geq 2$ labels, there exists no upper bound for the values of $m\left(N_{1}, N_{2}\right)$, as there exist arbitrarily large phylogenetic networks with $n$ leaves and no internal node of any one of them equivalent to an internal node of the other one. It remains open to compute the diameter of $m$ on restricted classes of phylogenetic networks, like, for instance, the reduced or the tree-child ones.

\section{ACKNOWLEDGMENTS}

The research described in this paper has been partially supported by the Spanish DGI projects MTM2006-07773 COMGRIO and MTM2006-15038-C02-01. The authors acknowledge with thanks the suggestions and comments of the reviewers, which have led to a substantial improvement of the paper.

\section{REFERENCES}

[1] M. Baroni, C. Semple, and M. Steel, "A Framework for Representing Reticulate Evolution," Annals of Combinatorics, vol. 8, no. 4, pp. 391-408, 2004.

[2] M. Baroni, C. Semple, and M. Steel, "Hybrids in Real Time," Systematic Biology, vol. 55, no. 1, pp. 46-56, 2006.

[3] G. Cardona, M. Llabrés, F. Rosselló, and G. Valiente, "A Distance Metric for a Class of Tree-Sibling Phylogenetic Networks," Bioinformatics, vol. 24, no. 13, pp. 1481-1488, 2008.

[4] G. Cardona, M. Llabrés, F. Rosselló, and G. Valiente, "Path Lengths in Tree-Child Time Consistent Hybridization Networks," http://arxiv.org/abs/0807.0087, 2008.

[5] G. Cardona, M. Llabrés, F. Rosselló, and G. Valiente, "Metrics for Phylogenetic Networks II: Nodal and Triplets Metrics," IEEE/ ACM Trans. Computational Biology and Bioinformatics, preprint, 21 Nov. 2008, doi: 10.1109/TCBB.2008.127.

[6] G. Cardona, M. Llabrés, F. Rosselló, and G. Valiente, "Tree-Sibling Time Consistent Phylogenetic Networks Are Graph IsomorphismComplete," http://arxiv.org/abs/0902.4640, 2009.

[7] G. Cardona, F. Rosselló, and G. Valiente, "Tripartitions Do Not Always Discriminate Phylogenetic Networks," Math. Biosciences, vol. 211, no. 2, pp. 356-370, 2008.

[8] G. Cardona, F. Rosselló, and G. Valiente, "Comparison of TreeChild Phylogenetic Networks," IEEE/ACM Trans. Computational Biology and Bioinformatics preprint, 3 Dec. 2007, doi: 10.1109/ TCBB.2007.70270.

[9] M.-M. Deza and E. Deza, Dictionary of Distances. Elsevier Science, 2006.

[10] A.J. Dobson, "Comparing the Shapes of Trees," Lecture Notes in Math., vol. 452, pp. 95-100, 1975.

[11] J. Felsenstein, Inferring Phylogenies. Sinauer Assoc., 2004.

[12] P. Flajolet, P. Sipala, and J.-M. Steyaert, "Analytic Variations on the Common Subexpression Problem," Proc. 17th Int'l Colloquium Automata, Languages, and Programming, pp. 220-234, 1990.

[13] G. Ganapathy, B. Goodson, R. Jansen, H. son Le, V. Ramachandran, and T. Warnow, "Pattern Identification in Biogeography," IEEE/ACM Trans. Computational Biology and Bioinformatics, vol. 3, no. 4, pp. 334-346, Oct.-Dec. 2006.

[14] K.T. Huber, B. Oxelman, M. Lott, and V. Moulton, "Reconstructing the Evolutionary History of Polyploids from Multilabeled Trees," Molecular Biology and Evolution, vol. 23, no. 9, pp. 17841791,2006
[15] E.M. Luks, "Isomorphism of Graphs of Bounded Valence Can Be Tested in Polynomial Time," J. Computer and System Sciences, vol. 25, no. 1, pp. 42-65, 1982.

[16] B.M.E. Moret, L. Nakhleh, T. Warnow, C.R. Linder, A. Tholse, A. Padolina, J. Sun, and R. Timme, "Phylogenetic Networks: Modeling, Reconstructibility, and Accuracy," IEEE/ACM Trans. Computational Biology and Bioinformatics, vol. 1, no. 1, pp. 13-23, Jan.-Mar. 2004.

[17] L. Nakhleh, "A Metric on the Space of Reduced Phylogenetic Networks," IEEE/ACM Trans. Computational Biology and Bioinformatics preprint, 6 Jan. 2009, doi: 10.1109/TCBB.2009.2.

[18] R. Page, "Maps between Trees and Cladistic Analysis of Historical Associations among Genes, Organisms, and Areas," Systematic Biology, vol. 43, no. 1, pp. 58-77, 1994.

[19] G. Valiente, Algorithms on Trees and Graphs. Springer, 2002.

[20] G. Valiente, "Efficient Algorithms on Trees and Graphs with Unique Node Labels," Applied Graph Theory in Computer Vision and Pattern Recognition, A. Kandel, H. Bunke, and M. Last, eds., vol. 52, pp. 137-149, Springer, 2007.

[21] S.M. Woolley, D. Posada, and K.A. Crandall, "A Comparison of Phylogenetic Network Methods Using Computer Simulation," Plos ONE, vol. 3, no. 4, p. e1913, 2008.

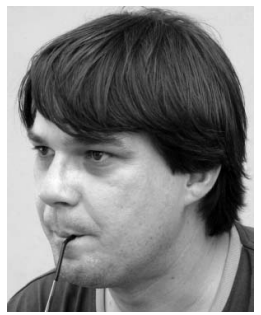

Gabriel Cardona is an associate professor in the Department of Mathematics and Computer Science, University of the Balearic Islands. He is a member of the Computational Biology and Bioinformatics Research Group at the University of the Balearic Islands, and the Number Theory Research Group at the Technical University of Catalonia. His research interests are split between number theory (mainly, arithmetical properties of curves of genus 2) and computational biology (mainly, mathematical models of evolution).

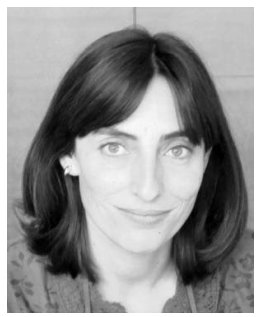

Mercè Llabrés is an associate professor in the Department of Mathematics and Computer Science and a member of the Computational Biology and Bioinformatics Research Group at the University of the Balearic Islands, Palma de Mallorca, Spain. Her research is centered on mathematical models in computational and systems biology.

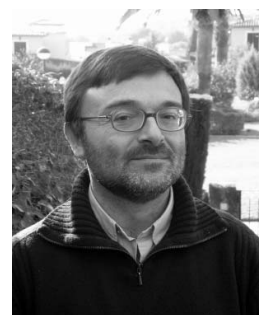

Francesc Rosselló is an associate professor in the Department of Mathematics and Computer Science, University of Balearic Islands, and the main researcher of the Computational Biology and Bioinformatics Research Group of the Research Insitute on Health Sciences (IUNICS) and the University of the Balearic Islands. His current research interests fall in the general areas of computational and systems biology, with emphasis on the development of new mathematical models in these areas and the comparison of graphs and other nonlinear structures arising in them.

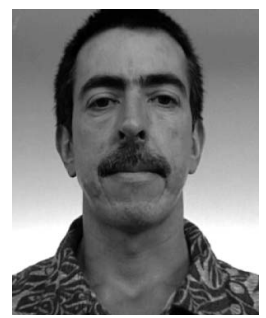

Gabriel Valiente is an associate professor in the Department of Software and a member of the Algorithms, Bioinformatics, Complexity and Formal Methods Research Group of the Technical University of Catalonia, Barcelona, Spain. $\mathrm{He}$ is also a member of the Computational Biology and Bioinformatics Research Group at the University of the Balearic Islands, and a permanent visiting professor at the Centre for Genomic Regulation in the Barcelona Biomedical Research Park. His research interests fall in the general areas of computational and systems biology, with emphasis on algorithms in bioinformatics and mathematical models in computational and systems biology. 